\title{
BMJ Open The impact of the Luton social prescribing programme on energy expenditure: a quantitative before-and- after study
}

\author{
Julia Vera Pescheny, ${ }^{1}$ Laura H Gunn, ${ }^{2,3}$ Gurch Randhawa, ${ }^{1}$ Yannis Pappas ${ }^{1}$
}

To cite: Pescheny JV, Gunn LH, Randhawa G, et al. The impact of the Luton social prescribing programme on energy expenditure: a quantitative before-andafter study. BMJ Open 2019;9:e026862. doi:10.1136/ bmjopen-2018-026862

- Pre-publication history and additional material is published online only. To view please visit the journal online (http://dx.doi org/10.1136/bmjopen-2018026862).

Received 23 September 2018

Revised 4 March 2019

Accepted 8 April 2019
D) Check for updates

(c) Author(s) (or their employer(s)) 2019. Re-use permitted under CC BY-NC. No commercial re-use. See rights and permissions. Published by BMJ.

${ }^{1}$ Institute of Health Research, University of Bedfordshire, Luton, UK

${ }^{2}$ Department of Public Health Sciences, University of North Carolina (UNC) at Charlotte, Charlotte, NC, USA

${ }^{3}$ School of Public Health, Imperial College London, London, UK

\section{Correspondence to} Mrs. Julia Vera Pescheny; julia.pescheny@study.beds. ac.uk

\section{ABSTRACT}

Objectives The objective of this study was to assess the change in energy expenditure levels of service users after participation in the Luton social prescribing programme.

Design Uncontrolled before-and-after study.

Setting This study was set in the East of England (Luton). Participants Service users with complete covariate information and baseline measurements $(n=146)$ were included in the analysis.

Intervention Social prescribing, which is an initiative that aims to link patients in primary care with sources of support within the community sector to improve their health, well-being and care experience. Service users were referred to 12 sessions (free of charge), usually provided by third sector organisations.

Primary outcome measure Energy expenditure measured as metabolic equivalent (MET) minutes per week.

Results Using a Bayesian zero-inflated negative binomial model to account for a large number of observed zeros in the data, 95\% posterior intervals show that energy expenditure from all levels of physical activities increased post intervention (walking 41.7\% (40.31\%, 43.11\%); moderate $5.0 \%$ (2.94\%, 7.09\%); vigorous $107.3 \%$ $(98.19 \%, 116.20 \%)$ and total $56.3 \%(54.77 \%, 57.69 \%))$. The probability of engaging in physical activity post intervention increased, in three of four MET physical activity levels, for those individuals who were inactive at the start of the programme. Age has a negative effect on energy expenditure from any physical activity level. Similarly, working status has a negative effect on energy expenditure in all but one MET physical activity level. No consistent pattern was observed across physical activity levels in the association between gender and energy expenditure.

Conclusion This study shows that social prescribing may have the potential to increase the physical activity levels of service users and promote the uptake of physical activity in inactive patient groups. Results of this study can inform future research in the field, which could be of use for commissioners and policy makers.

\section{INTRODUCTION}

Chronic conditions, such as type 2 diabetes, cardiovascular disease (CVD) and various respiratory diseases, create a serious public

\section{Strengths and limitations of this study}

- Employing zero-inflated methods to model the zero-inflated nature of the observed data allows for a more appropriate, unbiased analysis compared with prior approaches.

- This study is the first to evaluate effects of covariates (gender, age, working status) on energy expenditure in relation to social prescribing programmes.

- Use of the Bayesian approach allows for the information extracted to reflect the knowledge about the parameters of interest irrespective of small sample sizes.

- Implementing a Bayesian analysis facilitates a full representation of the distributions of the parameters of interest (oftentimes non-normal) as opposed to a normal approximation for the distributions.

- Similar to most social prescribing evaluations in the UK, this study lacks a control group and is limited by a high loss of follow-up.

health challenge in the UK and worldwide. ${ }^{1-3}$ It is estimated that the number of people living with multiple chronic conditions is steadily increasing in the $\mathrm{UK}^{4}$ The changing needs of the population increase costs and pressure on the UK's National Health Systems. However, most western healthcare systems are dominated by disease-oriented delivery, research and education (Biomedical model of care). ${ }^{1}$ It is clear that disease-oriented delivery of health services can no longer meet the complex needs of people with chronic conditions. ${ }^{5}$ Thus, the focus of health systems needs to shift from treatment of disease to primary, secondary and tertiary disease prevention, self-management and health behaviour and lifestyle modifications. ${ }^{15}$ In essence, the underlying model of care needs to change to a biopsychosocial model, considering, in addition to biological factors and medical interventions, the wider determinants of 
health and coordination and integration of care across professionals and disciplines. ${ }^{16}$

To respond to the changing needs of populations and increased pressure on the National Health Service (NHS), the integration of health and social care turned into a policy priority in the UK. ${ }^{7-9}$ Social prescribing is an increasingly implemented initiative in the UK, aiming to link primary care to non-clinical community services to promote self-management, health behaviour improvements and more effective responses to psychosocial problems (eg, social isolation, debt, housing and employment issues). ${ }^{10-12}$ There is no standard definition of social prescribing, but the National Social Prescribing Network defines it as "enabling healthcare professionals to refer patients to a link worker, to co-design a non-clinical social prescription to improve their health and wellbeing'. ${ }^{13}$ Typically, the role of link workers, also referred to as navigators, involve individual assessment to identify non-medical needs of service users and motivational interviewing, processing onward referrals to non-medical sources of support, providing continuous personalised support and collecting data for evaluations. ${ }^{10}$ Examples of sources of non-medical support, predominantly provided by third sector organisations, include art therapy, walking and reading groups, exercise and yoga classes, nature-based activities, volunteering, legal advice and support with employment, debt and housing. ${ }^{10} 14$

Most social prescribing evaluations are available from grey literature sources (eg, informal web-based sources and evaluation reports), and therefore often do not adhere to formal reporting and quality standards expected in academic journal articles. ${ }^{14-17}$ This trend may be explained by the fact that social prescribing is a relatively new field of research and that the 'scientific' evaluation of local schemes may be of minor interest and limited by restricted resources. ${ }^{17}$ However, there is emerging evidence that social prescribing has the potential to improve mental health (anxiety and depression), well-being, social isolation and to increase physical activity levels. ${ }^{18-21}$ Previous studies suggest evidence that regular physical activity reduces the risk of the development of chronic conditions, such as CVD, type 2 diabetes and cancer. $^{2322}$ In addition to primary prevention, physical activity improves quality of life and plays an important role in secondary prevention of chronic conditions by reducing the impact of the disease, slowing its progress and preventing recurrence. ${ }^{2} 2324$ Thus, the integration of primary care and physical activity services in the third sector to promote increased physical activity and exercise levels is important to meet the changing needs of populations. $^{2}$

The objective of this study was to assess the change in energy expenditure levels of service users after participation in a social prescribing programme. In addition, this study aims to evaluate the effects of covariates (age, gender, working status) on energy expenditure after engaging in the social prescribing service. Covariates help us describe the potential differences in proclivity, among clusters of individuals, to be affected by the intervention. Since we cannot assume a priori that all individuals will be equally affected by the intervention, we introduce covariates to explain any potential differences in activity changes among individuals. This is relevant because of the wide spectrum of individual's characteristics in the population of interest. Is there evidence that the intervention may induce a different effect for individuals with specific characteristics in terms of age or gender, for example? If so, those should be quantified.

Understanding the effects of covariates on energy expenditure can help to understand possible differences in energy expenditure between service user groups (eg, men and women or those working and not working). Differences in observed changes by service user groups may indicate that some groups require more support than others to engage in physical activity, and hence the results can inform service development. If there are no differences between groups, the resulting model is equivalent to one where the covariates were excluded a priori. Adding them to the initial model only enhances the potential explanatory power of the analysis. This study focuses on a social prescribing pilot programme that was implemented in 2015 in one Clinical Commissioning Group area in the East of England (Luton) across four general practices. The 3-year pilot programme was funded through the NHS Better Together Care Fund, which seeks to integrate health and social care services in England. The aim of the Luton social prescribing programme was to improve the health and well-being of service users, with a specific focus on physical activity and mental well-being. The pathway started with a referral from a general practitioner (GP) to a navigator. Based on the needs of the local community, the key target groups of the Luton social prescribing programme included people with high risk of or diagnosis with type 2 diabetes and COPD, people with mild to moderate mental health issues (particularly depression and anxiety), people who are experiencing loneliness and/or social isolation and carers. Although the specific focus was on the groups outlined above, GPs had the option to refer all patients who could benefit from social prescribing to the programme. To do so, the category 'Other' was included in the referral form to social prescribing. Following a referral, navigators then contacted referred patients to arrange an initial appointment held in surgeries. The role of navigators involved an individual assessment to identify the non-medical needs of service users, motivational interviewing, continuous personalised support and to link service users with non-medical sources of support, to help improve their health and well-being. The number of sessions with navigators and intensity of support provided were dependent on the individual's needs. In the Luton model, navigators could refer patients onwards to 12 sessions, usually provided by third sector organisations. Examples of such services include advice services (eg, debt, housing and employment), physical activities (eg, walking groups, aerobics and yoga), gardening, 


\begin{tabular}{|c|c|c|c|c|}
\hline $\begin{array}{c}\text { Patient } \\
\text { presents in } \\
\text { primary }\end{array}$ & $\begin{array}{l}\text { Healthcare } \\
\text { professional } \\
\text { identifies }\end{array}$ & $\begin{array}{c}\text { Navigator } \\
\text { contacts the } \\
\text { service user } \\
\text { for initial } \\
\text { assessment } \\
\end{array}$ & $\begin{array}{l}\text { Navigator } \\
\text { makes } \\
\text { an onward } \\
\text { referral, or } \\
\text { signposts }\end{array}$ & $\begin{array}{c}\text { Service } \\
\text { user attends } \\
\text { activities/ } \\
\text { services }\end{array}$ \\
\hline $\begin{array}{l}\text { care with } \\
\text { non- } \\
\text { medical } \\
\text { needs/ } \\
\text { psycho- } \\
\text { social } \\
\text { symptoms }\end{array}$ & $\begin{array}{l}\text { non-medical } \\
\text { needs and } \\
\text { makes a } \\
\text { referral to } \\
\text { social } \\
\text { prescribing }\end{array}$ & $\begin{array}{c}\text { Navigator } \\
\text { works with } \\
\text { service user } \\
\text { and assesses } \\
\text { his/her non- } \\
\text { medical needs }\end{array}$ & $\begin{array}{l}\text { service user } \\
\text { to non- } \\
\text { medical } \\
\text { sources of } \\
\text { support, } \\
\text { usually } \\
\text { provided } \\
\text { within the } \\
\text { third sector }\end{array}$ & \begin{tabular}{|} 
Re- \\
assessment \\
after a \\
number of \\
sessions \\
(usually 12) \\
and exit \\
interview
\end{tabular} \\
\hline
\end{tabular}

Figure 1 Luton social prescribing pathway.

social activities (eg, lunch clubs), stress management and relaxation courses and creative activities (eg, art clubs). Thus, although this study focuses on the changes in energy expenditure post intervention, referrals were also made to other services not related to physical activity. The 12 sessions were free of charge for service users. Individuals could continue to use the services beyond the social prescribing programme, but needed to cover the costs themselves. Figure 1 summarises the pathway of the Luton social prescribing model.

\section{METHODS}

\section{Setting and service}

This study was set in the East of England, in Luton. Luton is a city of around 200000 people with a large minority ethnic population. Luton is ranked as the 59th most deprived area in England by the Index of Multiple Deprivation, ranging from 1 (most deprived area) to 32844 (least deprived area in England). ${ }^{2526}$

\section{Design}

The design of this study is an uncontrolled before-andafter study.

\section{Data sources}

Navigators routinely collected data for referred patients in the first appointment (baseline) and in the last appointment (immediately post intervention). Data were collected for primary care patients referred to the Luton social prescribing programme between January 2016 and February 2018. Referrals to the social prescribing programme started in January 2016 and the pilot ended in March 2018. The routinely collected data were used for the analysis.

\section{Data cleaning}

Researchers from the University of Bedfordshire cleaned the data.

\section{Measure of energy expenditure}

The seven-item International Physical Activity Questionnaire (IPAQ) in English was used to collect data on physical activity levels of service users pre and immediately post intervention. The seven-item IPAQ covers the frequency and duration of walking, moderate and vigorous physical activities over the previous 7 days, and a single-item question on the duration of week-day sitting. ${ }^{27}$ In accordance with the officially recommended guidelines, the results of the IPAQ can be presented as a score in metabolic equivalent (MET) minutes per week for each activity category separately or as a sum. ${ }^{28}$ METs represent the energy expenditure of physical activities as a multiple of the resting metabolic rate. ${ }^{29}$ The following four formulas were used to calculate MET (minutes per week) from the seven-item IPAQ results in this study ${ }^{28}$ :

Walking MET (minutes/week) $=3.3 *$ walking minutes*walking days

Moderate MET $($ minutes $/$ week $)=4.0 *$ moderate intensity activity minutes*moderate intensity days

Vigorous MET (minutes $/$ week) $=8.0 *$ vigorous intensity activity minutes*vigorous intensity days

Total physical activity MET (minutes/week)=Walking MET (minutes/week)+ModerateMET (minutes/week)+Vigorous MET (minutes/week)

Minutes are reported in rounded integer value across all four levels of physical activity.

\section{Covariates}

The following three covariates were included in the analysis: Gender (male and female), age (continuous variable) and working status (working and not working). The categories working, self-employed, carer and individuals having any of these options were grouped into a single category, referred to as working. The categories unemployed, home maker, retired, student, disabled and long-term sick were grouped into another category, and referred to as not working.

\section{Statistical analysis}

Data were analysed using the SPSS V.23 and an open source Bayesian inferential software called OpenBugs. Frequencies and descriptive statistics (mean, SD and range) were used to determine participant characteristics and to examine the distribution of the outcome variable.

The distributions of the variables Walking, Moderate, Vigorous and Total MET (all in minutes per week) were positively skewed and overdispersed. In addition, there was a high prevalence of zeros in the data, especially for Moderate and Vigorous MET (minutes per week). The presence of a large number of zeros in datasets creates challenges for analysis, as they cannot be justified by standard distributions and may prevent the transformation of data in an attempt to meet distributional (eg, normal) assumptions.

We used the Bayesian zero-inflated negative binomial model to assess the changes in energy expenditure before and after participation in the Luton social prescribing programme. We model the probabilities of non-zero MET scores as

$$
\begin{aligned}
\operatorname{logit}\left(p_{i, t}\right)= & \beta_{0}^{*}+\beta_{1}^{*} * \text { AGE }_{i}+\beta_{2}^{*} * \text { GENDER }_{i} \\
& +\beta_{3}^{*} * \text { EMPLOYMENT } \\
i & +\theta^{*} * t
\end{aligned}
$$


and the mean of non-zero MET scores as

$$
\begin{aligned}
\log \left(\mu_{\mathrm{i}, \mathrm{t}}^{*}\right)= & \beta_{0}+\beta_{1} * A G E_{i}+\beta_{2} * \text { GENDER }_{i} \\
& +\beta_{3} * \text { EMPLOYMENT }_{i}+\theta * t
\end{aligned}
$$

where $i$ indicates the individual, $t$ indicates time ( $t=0$ is pre intervention and $t=1$ is post intervention), $\beta_{k}$ and $\beta_{k}^{*}(\mathrm{k}=1,2,3)$ indicate the effect of the included covariates (ie, age, gender and working status) on the non-zero mean MET score $\left(\mu_{i, t}^{*}\right)$ and on the probability of a non-zero MET score $\left(p_{i, t}\right)$ respectively, $\theta$ represents the common percent change in mean non-zero score $\left(\mu_{i, t}^{*}\right)$ due to the intervention, and $\theta^{*}$ indicates the effect of the intervention on the probability of having a non-zero value. Since the effect of the intervention on the probability of non-zero scores $\left(\theta^{*}\right)$ is not constant across individuals (due to the logistic link), we provide instead summaries of the posterior distribution of the changes in probability due to the intervention averaged over individuals. A different model was fit for each of the four MET scores, and the results are reported for each of the four models.

The Bayesian specification is completed in the following form:

$$
\begin{gathered}
\operatorname{MET}_{i, t} \sim\left\{\begin{array}{l}
N B\left(\mu_{i, t}^{*}, \sigma_{i, t}\right), \text { with probability } p_{i, t} \\
0, \text { with probability } 1-p_{i, t}
\end{array}\right. \\
\beta_{k}, \beta_{k}^{*}, \theta, \theta^{*} \sim N(0, \boldsymbol{\zeta I}),
\end{gathered}
$$

where the parametrisation for the negative binomial as a function of its mean takes the form

$$
Y \sim N B(\mu, \sigma) \equiv P(Y=m)=\left(\begin{array}{c}
m-1+\frac{\mu^{2}}{\sigma^{2}-\mu} \\
m^{2}
\end{array}\left(\frac{\sigma^{2}-\mu}{\sigma^{2}}\right)^{m}\left(\frac{\mu}{\sigma^{2}}\right)^{\frac{\mu^{2}}{\sigma^{2}-\mu}},\right.
$$

and the priors for the remaining parameters follow independent normal distributions with standard parametrisation and hyperparameter vector of variances $\zeta$, with $\boldsymbol{I}$ as the identity matrix.

Zero-inflated models ${ }^{30} 31$ allow for expansion of traditional count models, such as Poisson or negative binomial, to account for excess zeros due to structural reasons. These models are of natural applicability to physical activity, where there are two major states of nature, one on and one off: some physical activity or no physical activity (zero). A negative binomial approach is appropriate for cases of overdispersed distributions (SD of the data significantly larger than the overall mean), which is the case in non-zero physical activity across a wide spectrum of individuals' baseline characteristics.

The choice of Bayesian methods was made for two reasons: (1) low sample size presented in this study relative to the parametric complexity of the model (Bayesian methods do not rely on asymptotic theory to produce outcomes) and (2) built-in capacity to introduce prior information, which, although not relevant within this study (we use non-informative priors), would be more relevant when combining the outcomes in this manuscript with future research in this area (eg, informing future priors).

Given that we have no prior knowledge on service users' MET (minutes per week) obtained from the seven-item IPAQ pre and post participation in social prescribing, standard flat priors (non-informative) were used for the gamma hyperparameters in the gamma-poisson parametrization of the negative binomial, as well as the remaining parameters of the model. ${ }^{32}$ A sensitivity analysis was conducted where a wide range of hyperparameters $(\zeta)$ was tested, yielding similar posterior results, so the final specification is based on $\zeta_{\mathrm{k}}=10^{6}$. A total of 200000 samples were drawn from the posterior distribution for each of the four MET models, with a 10000 iteration burn-in and the Markov Chain Monte Carlo (MCMC) thinned to keep every 10th draw to address very minor autocorrelation. The MCMC mixed appropriately and the goodness of fit tests indicate good fit of the model. ${ }^{33} 34$ The negative binomial model was fit using a more efficient parametrisation (as a function of the mean), through a gamma mixture of Poisson distributions. ${ }^{35}$ This parametrisation allows for fast and efficient mixing of the chain.

\section{Patient and public involvement}

Patients were not involved in the development of the study design.

\section{RESULTS \\ Descriptive findings}

In total, 448 patients were referred to the social prescribing programme and included in the database from January 2016 to February 2018. Of these, 186 individuals (41.5\%) completed the baseline assessment with the seven-item IPAQ and 56 (30.1\% of those at baseline) completed the follow-up immediately post intervention (table 1 ). Thus, the majority of participants $(70 \%)$ were lost to follow-up or did not engage with the social prescribing service after the initial assessment and hence did not complete post-measures. Significant loss to follow-up was frequently reported in previous studies on social prescribing programmes in the UK. ${ }^{15}{ }^{36}$ Table 2 demonstrates that the percent of zero response was lower at follow-up at all activity levels, compared with the baseline. In addition,

Table 1 Available data on energy expenditure pre and post intervention $(n=448)$

\begin{tabular}{ll}
\hline $\begin{array}{l}\text { MET minutes per week (seven-item } \\
\text { IPAQ) }\end{array}$ & $\mathbf{n}(\%)$ \\
\hline Baseline & $186(41.5)$ \\
Data missing & $262(58.5)$ \\
Total & $448(100)$ \\
Post intervention & $56(12.5)$ \\
Data missing & $392(87.5)$ \\
Total & $448(100)$ \\
\hline
\end{tabular}

IPAQ, International Physical Activity Questionnaire; MET, metabolic equivalent. 
Table 2 Descriptive outcomes for Walking, Moderate, Vigorous and Total (MET minutes per week) at baseline and follow-up

Walking (MET minutes per week) $\%$ of zero response, mean (SD) of non-zero response

\begin{tabular}{lll}
\hline $\begin{array}{l}\text { Baseline } \\
(n=186)\end{array}$ & $36.6,714.5(1266.2)$ & $79.6,554.4(679.2)$ \\
\hline $\begin{array}{l}\text { Follow-up } \\
(n=56)\end{array}$ & $14.3,1163.3(1449.1)$ & $53.6,558.5(495.8)$ \\
\hline
\end{tabular}

Moderate activity (MET minutes per week) $\%$ of zero response, mean (SD) of non-zero response

Vigorous activity (MET
minutes per week)
$\%$ of zero response,
mean (SD) of non-zero
response

$96.8,508$ (496.4)
Total physical activity (MET minutes per week) $\%$ of zero response, mean (SD) of non-zero response

30.1, 831.1 (1325.8)

$7.1,1477.6(1573.9)$

MET, metabolic equivalent.

the mean MET minutes per week increased post intervention across all activity levels, compared with the baseline.

The baseline characteristics of the participants are shown in table 3 . The mean age of service users was 51.2 (SD 15.7) years, and $70.4 \%$ were female. The majority of service users were not working $(61.8 \%)$. Data on working status are missing for $21 \%$ of the sample.

\section{Bayesian analysis}

Individuals with complete covariate information and baseline measurements only $(n=146)$ were included in the Bayesian analysis. For simplicity across all analyses, interpretations of results focus on assessing evidence of directionality in relationships tested. In order to summarise those interpretations homogeneously across measures (ie, Walking, Moderate, Vigorous and Total MET scores), conclusions are built on 95\% posterior intervals. Evidence of relationships is reported when at least $97.5 \%$ of the posterior density falls in either positive or negative territory (ie, when the $95 \%$ equal-tailed posterior probability interval (PPI) reported is bounded by values with the same sign). Unclear evidence of a relationship is reported in all other cases. In a more purist

Table 3 Sociodemographic characteristics of the sample $(n=186)$ for baseline data collected with the seven-item IPAQ and proportions of missing data

\begin{tabular}{ll}
\hline Characteristics & $\mathbf{n}(\mathbf{\%})$ \\
\hline Sex & \\
Female & $131(70.4)$ \\
Male & $55(29.6)$ \\
Data missing & $0(0.0)$ \\
Total & $186(100)$ \\
Working status & \\
Working & $32(17.2)$ \\
Not working & $115(61.8)$ \\
Data missing & $39(21.0)$ \\
Total & $186(100)$ \\
& Mean (SD), missing data $\mathbf{n ~ ( \% ) ~}$ \\
& (n=186) \\
Age & $51.2(15.7), 1(0.5)$ \\
\hline
\end{tabular}

IPAQ, International Physical Activity Questionnaire.
Bayesian interpretation, the parameters of interest are random variables, and reported posterior distributions simply represent our posterior knowledge about them. Therefore, we define 'evidence' in this manuscript when (at least) $97.5 \%$ posterior probability of the parameter of interest shares a common sign. However, all evidence reported is conditional on the before-and-after study design, including its limitations (see Discussion section).

\section{Walking MET (minutes per week)}

The expected increase in the mean non-zero Walking MET score due to the intervention is approximately $41.7 \%$ (95\% PPI $40.31 \%, 43.11 \%$ ). The average increase in the probability of observing non-zero Walking MET scores due to the intervention is $25.2 \%$ (95\% PPI $11.66 \%$, $37.37 \%)$.

There seems to be a negative effect of age on the mean non-zero Walking MET score (table 4). This indicates that the older the individual, the lower will be the expected Walking MET score. Similarly, there is evidence for a positive effect of gender on the mean non-zero Walking MET score. Thus, males have a higher expected non-zero Walking MET score than females. Lastly, there is a negative effect of working on the mean non-zero Walking MET score. This indicates that those individuals who were classified as working have a lower expected non-zero Walking MET score than those who were classified as not working.

There was weak or no evidence of the effect of age, gender and working status on the probability of observing a non-zero Walking MET score. The results for the MET scores from walking are summarised in table 4 .

\section{Moderate MET (minutes per week)}

The expected increase in the mean non-zero Moderate MET score due to the intervention is $5.0 \%$ (95\% PPI $2.94 \%, 7.09 \%$ ). The average increase in the probability of observing non-zero Moderate MET scores due to the intervention is $23.5 \%$ (95\% PPI 9.05\%, 38.08\%).

Similar to Walking MET minutes per week, there is evidence for a negative effect of age on the mean non-zero Moderate MET score (table 5). In contrast to Walking MET minutes per week, there is evidence for a negative effect of gender on the mean non-zero Moderate MET score. This indicates that males have a lower expected 
Table 4 Summary of results for Walking MET scores

\section{Outcomes}

Mean (\%) (95\% posterior probability interval)

Common percent (\%) increase in mean non-zero Walking MET score $(\theta)$

Evidence for positive effect $41.7(40.31,43.11)$

Average increase in the probability of observing non-zero Walking MET scores

\begin{tabular}{|c|c|}
\hline Effects of covariates & $\begin{array}{l}\text { Direction of evidence } \\
\text { Mean effect ( } 95 \% \text { posterior probability interval) }\end{array}$ \\
\hline Effect of age on the mean non-zero Walking MET score $\left(\beta_{1}\right)$ & Evidence for negative effect $-0.006(-0.0067,-0.0059)$ \\
\hline Effect of gender* on the mean non-zero Walking MET score $\left(\beta_{2}\right)$ & Evidence for positive effect $0.38(0.37,0.39)$ \\
\hline $\begin{array}{l}\text { Effect of gender }{ }^{*} \text { on the probability of observing non-zero Walking } \\
\text { MET scores }\left(\beta_{2}^{*}\right)\end{array}$ & Unclear evidence for effect $0.15(-0.54,0.84)$ \\
\hline $\begin{array}{l}\text { Effect of working status } † \text { on the mean non-zero Walking MET score } \\
\left(\beta_{3}\right)\end{array}$ & Evidence for negative effect $-0.11(-0.12,-0.097)$ \\
\hline $\begin{array}{l}\text { Effect of working status }{ }^{\dagger} \text { on the probability of observing non-zero } \\
\text { Walking MET scores }\left(\beta_{3}{ }^{*}\right)\end{array}$ & Unclear evidence for effect $-0.75(-1.60,0.058)$ \\
\hline
\end{tabular}

*The gender component is the incremental effect of being male on the mean non-zero MET scores and corresponding probabilities of nonzero MET scores.

†The working status component is the incremental effect of working on the mean non-zero MET scores and corresponding probabilities of non-zero MET scores.

MET, metabolic equivalent.

non-zero Moderate MET score than females. There is no clear indication of an impact of working on the mean non-zero Moderate MET score.
There is evidence for a negative effect of age on the probability of observing non-zero Moderate MET scores. This indicates that the higher the age of an individual,

Table 5 Summary of results for Moderate MET scores

Outcomes Mean (\%) (95\% posterior probability interval)

Common percent (\%) increase in mean non-zero Moderate Evidence for positive effect $5.0(2.94,7.09)$

MET score $(\theta)$

Average increase in the probability of observing non-zero Evidence for positive effect $23.5(9.05,38.08)$

Moderate MET scores

\section{Effects of covariates}

\section{Direction of evidence} Mean effect (95\% posterior probability interval)

Effect of age on the mean non-zero Moderate MET score $\left(\beta_{1}\right)$

Evidence for negative effect $-0.01(-0.011,-0.0089)$

Effect of age on the probability of observing non-zero Evidence for negative effect $-0.03(-0.057,-0.012)$ Moderate MET scores $\left(\beta_{1}^{*}\right)$

Effect of gender* on the mean non-zero Moderate MET Evidence for negative effect $-0.45(-0.48,-0.42)$ score $\left(\beta_{2}\right)$

\section{Effect of gender on the probability of observing non-zero} Moderate MET scores $\left(\beta_{2}^{*}\right)$

Effect of working status $\dagger$ on the mean non-zero Moderate Unclear evidence for effect $0.03(-0.00014,0.053)$

MET score $\left(\beta_{3}\right)$
Effect of working status ${ }^{\dagger}$ on the probability of observing
Unclear evidence for effect $0.52(-0.27,1.34)$ non-zero Moderate MET scores $\left(\beta_{3}{ }^{*}\right)$

${ }^{*}$ The gender component is the incremental effect of being male on the mean non-zero MET scores and corresponding probabilities of nonzero MET scores.

†The working status component is the incremental effect of working on the mean non-zero MET scores and corresponding probabilities of non-zero MET scores.

MET, metabolic equivalent. 
Table 6 Summary of results for Vigorous MET scores

\section{Outcomes}

Mean (\%) (95\% posterior probability interval)

Common percent (\%) increase in mean non-zero Vigorous MET score

Evidence for positive effect $107.3(98.19,116.20)$

$(\theta)$

Average increase in the probability of observing non-zero Vigorous MET Unclear evidence for effect $6.0(-1.21,15.07)$ scores

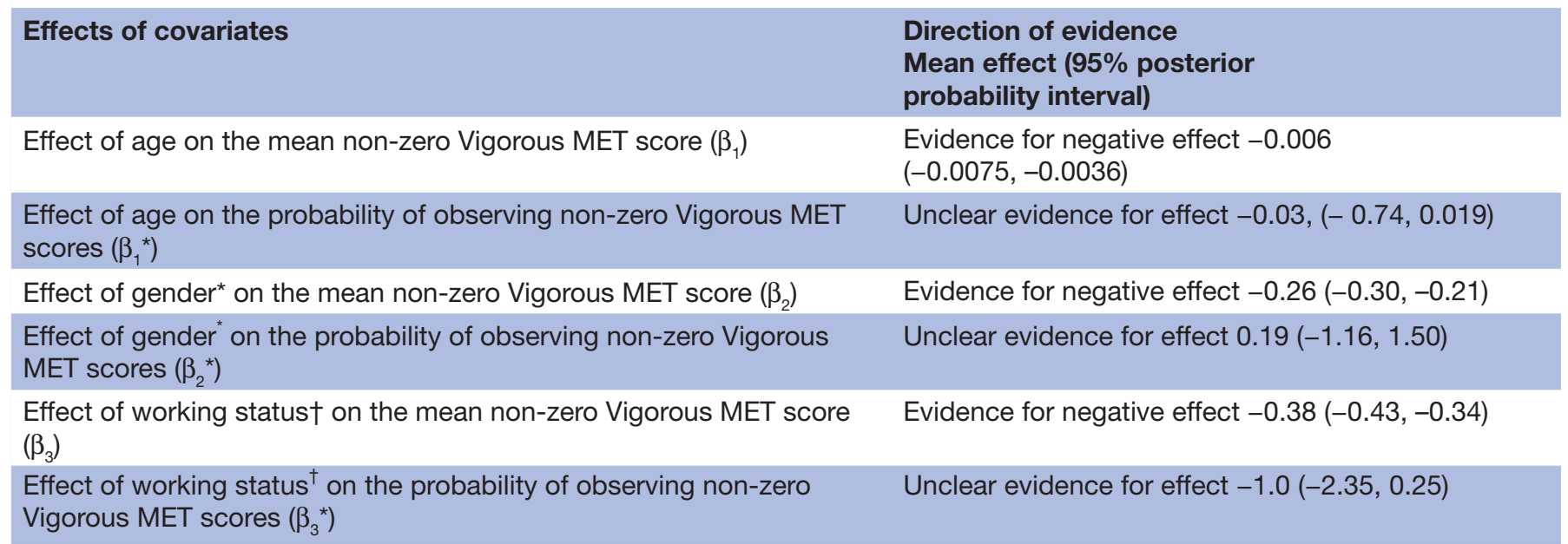

${ }^{*}$ The gender component is the incremental effect of being male on the mean non-zero MET scores and corresponding probabilities of nonzero MET scores.

†The working status component is the incremental effect of working on the mean non-zero MET scores and corresponding probabilities of non-zero MET scores.

MET, metabolic equivalent.

the lower will be the probability of observing non-zero Moderate MET scores. There were no and weak evidence for the effect of gender and working status on the probability of observing non-zero Moderate MET scores, accordingly. Thus, those individuals who were classified as working may have a higher probability of non-zero Moderate MET scores compared with those not working. The results for the MET scores from moderate physical activity are summarised in table 5 .

\section{Vigorous MET (minutes per week)}

The expected increase in the mean non-zero Vigorous MET score due to the intervention is $107.3 \%$ (95\% PPI $98.19 \%, 116.20 \%)$. The average increase in the probability of observing non-zero Vigorous MET scores due to the intervention is $6.0 \%$ (95\% PPI $-1.21 \%, 15.07 \%$ ).

There is evidence for a negative effect of age on the mean non-zero Vigorous MET scores (table 6). This negative effect indicates that the older an individual, the lower will be the expected non-zero Vigorous MET score. There also appears to be evidence for a negative effect of gender on the mean non-zero Vigorous MET scores. Thus, being male has an association with lower expected Vigorous MET scores than being female. Lastly, there is evidence for a negative effect of working on the mean non-zero Vigorous MET scores, indicating that those who are working have a lower expected non-zero Vigorous MET score than those who are not working.

There is unclear evidence for the effect of age, gender and working status on the probability of observing non-zero Vigorous MET scores. The results of MET scores for vigorous physical activity are summarised in table 6 .

\section{Total MET (minutes per week)}

The expected increase in the mean non-zero Total MET score due to the intervention is $56.3 \%$ (95\% PPI $54.77 \%$, $57.69 \%$ ), and the change is clearly positive. The average increase in the probability of observing a non-zero Total MET score due to the intervention is $26.4 \%$ (95\% PPI $14.98 \%, 36.40 \%)$, and the change is clearly positive.

There is evidence for a negative effect of age on the mean non-zero Total MET score (table 7), indicating that the older an individual, the lower will be the expected mean non-zero Total MET score. There is a positive effect of gender on the mean non-zero Total MET score, indicating that men have a higher expected mean non-zero Total MET score than women. Lastly, there is a negative effect of working on the mean non-zero Total MET score. This indicates that individuals who were classified as working have a lower expected non-zero Total MET mean score than those individuals who were classified as not working.

There is unclear evidence for the effect of age, gender and working status on the probability of observing non-zero Total MET scores. The results of MET scores for Total MET are summarised in table 7 .

\section{DISCUSSION}

The results of this study show evidence that the levels of energy expenditure (MET minutes/week) from walking, 
Table 7 Summary of results for Total MET scores

Outcomes Mean (\%) (95\% posterior probability interval)

Common percent (\%) increase in mean non-zero Total Evidence for positive effect $56.3(54.77,57.69)$ MET score $(\theta)$

Average increase in the probability of observing non- Evidence for positive effect $26.4(14.98,36.40)$

zero Total MET scores

$\begin{array}{ll}\text { Effects of covariates } & \text { Direction of evidence } \\ & \text { Mean effect ( } 95 \% \text { posterior } \\ & \text { probability interval) }\end{array}$

Effect of age on the mean non-zero Total MET score Evidence for negative effect $-0.01(-0.012,-0.011)$

$\left(\beta_{1}\right)$

Effect of age on the probability of observing non-zero Unclear evidence for effect $-0.01(-0.035,0.0097)$

Total MET scores $\left(\beta_{1}^{*}\right)$

Effect of gender* on the mean non-zero Total MET Evidence for positive effect $0.09(0.080,0.10)$

score $\left(\beta_{2}\right)$

Effect of gender ${ }^{*}$ on the probability of observing non- Unclear evidence for effect $0.74(-0.039,1.56)$

zero Total MET scores $\left(\beta_{2}^{*}\right)$

Effect of working status $\dagger$ on the mean non-zero Total Evidence for negative effect $-0.08,(-0.087,-0.067)$

MET score $\left(\beta_{3}\right)$

Effect of working status ${ }^{\dagger}$ on the probability of $\quad$ Unclear evidence for effect $-0.82,(-1.81,0.087)$

observing non-zero Total MET scores $\left(\beta_{3}{ }^{*}\right)$

${ }^{*}$ The gender component is the incremental effect of being male on the mean non-zero MET scores and corresponding probabilities of nonzero MET scores.

†The working status component is the incremental effect of working on the mean non-zero MET scores and corresponding probabilities of non-zero MET scores.

MET, metabolic equivalent.

moderate and vigorous physical activity may be increased through the intervention. Previous studies suggest evidence that small increases in energy expenditure (100-200 kcal/ day) may be sufficient to prevent gradual weight gain in adults. ${ }^{37-39}$ Previous studies identified that working with a navigator is a key enabler of behaviour change in service users. ${ }^{10} 19$ 40-42 Ongoing support, motivation and the development of open and trusting relationships appeared to improve the self-concept and independence of service users and made them feel prepared and ready to participate in the referred activities. ${ }^{10}$ Additionally, motivation, as well as continuous and need-driven support, from navigators appeared to promote adherence to activities, which is likely to affect behaviour change and service user outcomes. ${ }^{10}$ Based on these findings, navigators appear to be a key component of the social prescribing pathway, and may partly explain how and why social prescribing interventions work. ${ }^{43}$ Previous qualitative research on the Luton social prescribing programme has found that the navigators play a key role in promoting the uptake and adherence to referred activities, particularly physical activities. ${ }^{44}$ Thus, it is likely that the navigators' support contributed to the uptake and continuous engagement in physical activities, and in turn the observed increases in energy expenditure. However, it remains unclear whether the observed increase in energy expenditure remains on a longer term. Although previous research on the Luton social prescribing programme indicates that free services may promote uptake of social prescribing, ${ }^{44}$ it is possible that free services lead to the discontinuation of the adopted behaviour, if individuals cannot afford to continue activities beyond the social prescribing service. This issue may be particularly relevant when social prescribing is implemented in areas with high deprivation levels, such as Luton. Previous research found that financial incentives (eg, free gym memberships) to increase physical activity levels in adults were effective only on the short term, as after ending the incentive, the adopted physical activity behaviour was discontinued. $^{45}$

This is the first study to evaluate the trends between covariates (gender, age, working status) and energy expenditure in relation to social prescribing programmes. Therefore, it adds to the sparse knowledge of the effect of covariates on service user outcomes of social prescribing. The study found a negative association between age and energy expenditure from any physical activity level (ie, older individuals have lower expected MET scores). This finding is in line with what was expected, as previous research found that with ageing, the value of MET scores significantly reduces $(\mathrm{p}<0.05){ }^{46}$ Similarly, working had a negative effect on energy expenditure in all but one MET level (ie, individuals who were classified as working tend to have lower expected MET scores, though evidence was unclear for moderate physical activity). This finding indicates that it may be more difficult for service users who are working to engage in physical activities than for those who are not working. This trend may be explained by a lack of time to engage in physical activities during the day. Thus, it may be beneficial to ensure that social prescribing activities are available for service users before and after working hours and at the weekend. No consistent pattern was observed across physical activity 
levels in the association between gender and energy expenditure (ie, males have higher expected Walking and Total MET scores, whereas females have higher Moderate and Vigorous MET scores). In addition, this study found that the social prescribing programme might have the potential to promote physical activity in inactive patient groups. However, it is important to note that the outcomes of complex interventions, such as social prescribing, can be influenced by the implementation process, contextual factors, delivery models, pathways and individuals involved in the implementation and delivery. ${ }^{43}{ }^{44}$ Therefore, results of this study may not be generalisable across settings, populations and social prescribing programmes. Nevertheless, results of this study can provide important information and guidance for policy makers, commissioners and providers planning to implement, or upscale, social prescribing within primary care.

Some methodological strengths ${ }^{47}$ of the Bayesian approach include (1) the information extracted reflects the knowledge about the parameters of interest irrespective of small sample sizes; (2) the model allows for prior information (if/when available) to be incorporated into the analysis seamlessly, providing a framework for representation of cumulative information and (3) a full representation of the distributions of the parameters of interest (oftentimes non-normal) as opposed to a normal approximation for the distributions. This study has important limitations to consider. Similar to most evaluations of social prescribing programmes in the UK, this study is limited by missing data, high loss of follow-up, and a short follow-up period (ie, immediately post intervention). ${ }^{1536}$ Many of these limitations can be explained by the experienced challenges of developing and implementing a shared information technology (IT) system to facilitate communication between front-line providers, the collection of complete and standardised data and data sharing. At the end of the pilot period, the Luton team was still attempting to work around issues with information governance, security and feasibility of data sharing between providers. However, the development of shared IT systems between the health and social care sector was identified as a barrier in other integrated care programmes too. ${ }^{49}{ }^{50}$ Significant delays in the development of the shared IT system hindered the collection of standardised data. Although data on the ethnicity of service users, types of referred services and activities, number of attended sessions, adherence to the service and dropouts were initially planned to be included in the analysis, it was not possible due to the lack of standardised reporting and incomplete data. Thus, these covariates could not be included in the present analysis, but might be of interest in future studies to assess who received what, for what duration and with what effect. Another limitation of this before-and-after study is the lack of a control group. It was not possible to compare outcomes from those engaging with the service with those who did not, due to practical and resource issues. Thus, the results of this before-and-after study need to be interpreted with caution, as it is possible that the observed differences are attributed to other factors, rather than the engagement in the social prescribing service. In order to strengthen the evidence base on social prescribing and overcome these common methodological limitations in future studies, the development of evaluation frameworks at an early planning stage, sufficient resources and the supporting technological infrastructure are required.

Previous evaluations assessing the change in physical activity levels of social prescribing service users post intervention found mixed results. ${ }^{19} 51$ The evaluation by Wigfield et al found no statistically significant change in 'Minutes walking a day', 'Minutes of hard breathing exercise per week' and 'Minutes of muscle strengthening activity per week' at 3-months follow-up. ${ }^{51}$ The study by Kimberlee $e t$ al found a statistically significant increase $(\mathrm{p}<0.05)$ in the frequency of mean days on which individuals (1) walk more than 10 minutes and (2) do moderate exercise for $30 \mathrm{~min}$, at 3-months follow-up (27-item IPAQ). ${ }^{19}$ No statistically significant change was observed for the frequency of vigorous physical activities between baseline and follow-up mean day scores. ${ }^{19}$ Although the study by Kimberlee et aland the current study found an increase in walking and moderate exercises post intervention, results cannot be directly compared due to different outcome measures (mean day scores vs MET minutes per week) and methods of analysis. ${ }^{19}$ This variety in outcome measures and research methods between the previous studies may also explain the mixed evidence base. In line with our results, previous qualitative studies found that service users experienced an increase in their physical activity levels since being referred to social prescribing. ${ }^{10} 1840$

\section{CONCLUSION}

This study has shown that social prescribing may have the potential to increase the physical activity levels of service users and promote the uptake of physical activity in inactive patient groups. The use of Bayesian modelling does not need to rely on large samples and can incorporate the uncertainty embedded in a low sample setting. Additionally, it incorporates the information contained regarding individuals who were lost to follow-up, from which information borrowing is possible to learn about some of the common parameters. Results of this study can inform future research in the field, which could be of use for commissioners and policy makers.

Contributors YP and GR developed the initial protocol for the study. LHG and JVP have conducted the analysis and interpreted the data. JVP wrote the first draft of the paper. All authors (JVP, LHG, GR and YP) revised the paper critically and approved the final version to be published. All the authors (JVP, LHG, GR and YP) are responsible for the study concept and design.

Funding This study is part of a larger mixed methods doctorate study (20152018), which was funded by the Luton Borough Council.

Competing interests None declared.

Patient consent for publication Not required.

Ethics approval This research has been reviewed and given favourable opinion by the Institute for Health Research Ethics Committee at the University of Bedfordshire on 16 November 2016.

Provenance and peer review Not commissioned; externally peer reviewed.

Data sharing statement This study was conducted as part of a PhD degree at the University of Bedfordshire, and we do not have the approval to make the data publicly available. 
Open access This is an open access article distributed in accordance with the Creative Commons Attribution Non Commercial (CC BY-NC 4.0) license, which permits others to distribute, remix, adapt, build upon this work non-commercially, and license their derivative works on different terms, provided the original work is properly cited, appropriate credit is given, any changes made indicated, and the use is non-commercial. See: http://creativecommons.org/licenses/by-nc/4.0/.

\section{REFERENCES}

1. Barnett K, Mercer SW, Norbury M, et al. Epidemiology of multimorbidity and implications for health care, research, and medical education: a cross-sectional study. Lancet 2012;380:37-43.

2. Durstine JL, Gordon B, Wang Z, et al. Chronic disease and the link to physical activity. J Sport Health Sci 2013;2:3-11.

3. Marques A, Santos T, Martins J, et al. The association between physical activity and chronic diseases in European adults. Eur $J$ Sport Sci 2018;18:140-9.

4. Department of Health. Long term conditions compendium of information. Third edition, 2012:1-55. https://www.gov.uk/ government/uploads/system/uploads/attachment_data/file/216528/ dh_134486.pdf. (accessed 3 Jul 2018).

5. Eaton S, Roberts S, Turner B. Delivering person centred care in long term conditions. BMJ 2015;350:h181.

6. Farre A, Rapley T. The New Old (and Old New) Medical Model: Four Decades Navigating the Biomedical and Psychosocial Understandings of Health and IIIness. Healthcare 2017;5:88.

7. NHS England. Five Year Forward View. 2014 https://www.england. nhs.uk/ourwork/futurenhs/nhs-five-year-forward-view-web-version/ (accessed 5 Apr 2018).

8. NHS England. General Practice Forward View. 2016 https://www. england.nhs.uk/wp-content/uploads/2016/04/gpfv.pdf (accessed 5 Apr 2018).

9. Chatterjee HJ, Camic PM, Lockyer B, et al. Non-clinical community interventions: a systematised review of social prescribing schemes. Arts Health 2017;3015:1-27.

10. Moffatt S, Steer M, Lawson S, et al. Link Worker social prescribing to improve health and well-being for people with long-term conditions: qualitative study of service user perceptions. BMJ Open 2017;7:e015203.

11. Brandling J, House W. Social prescribing in general practice: adding meaning to medicine. Br J Gen Pract 2009;59:454-6.

12. Loftus AM, McCauley F, McCarron MO. Impact of social prescribing on general practice workload and polypharmacy. Public Health 2017;148:96-101.

13. Polley M, Dixon M, Pilkington K, et al. Report of the Annual Social Prescribing Network Conference. 2016:1-48 http://www. artshealthresources.org.uk/docs/report-of-the-inaugural-socialprescribing-network-conference/ (accessed 18 Oct 2018).

14. Centre for Reviews and Dissemination. Evidence to inform the commissioning of social prescribing. 2015;8 https://www.york.ac.uk/ media/crd/Evbriefing_social_prescribing.pdf.

15. Bickerdike L, Booth A, Wilson PM, et al. Social prescribing: less rhetoric and more reality. A systematic review of the evidence. BMJ Open 2017;7:e013384

16. Pescheny JV, Pappas Y, Randhawa G. Facilitators and barriers of implementing and delivering social prescribing services: a systematic review. BMC Health Serv Res 2018;18:86.

17. Pilkington K, Loef M, Polley M. Searching for Real-World Effectiveness of Health Care Innovations: Scoping Study of Social Prescribing for Diabetes. J Med Internet Res 2017;19:e20

18. Dayson C, Bashir N, Bennett E, et al. The Rotherham Socia Prescribing Service for People with Long-Term Health Conditions: Annual Evaluation Report. 2016:1-67 https://www4.shu.ac.uk/ research/cresr/sites/shu.ac.uk/files/rotherham-social-prescribingannual-eval-report-2016_7.pdf (accessed 16 Mar 2018).

19. Kimberlee R, Ward R, Jones $M$, et al. Measuring the economic impact of Wellspring Healthy Living Centre's social prescribing wellbeing programme for low level mental health issues encountered by GP services. 2014;111 http://www.wellspringhlc.org.uk/reports/ POV_Final_Report_March_2014.pdf.

20. Morton L, Ferguson M, Baty F. Improving wellbeing and self-efficacy by social prescription. Public Health 2015;129:286-9.

21. Grant C, Goodenough T, Harvey I, et al. A randomised controlled tria and economic evaluation of a referrals facilitator between primary care and the voluntary sector. BMJ 2000;320:419-23.

22. Booth FW, Roberts CK, Laye MJ. Lack of exercise is a major cause of chronic diseases. Compr Physiol 2012;2:1143-211.

23. Alves AJ, Viana JL, Cavalcante SL, et al. Physical activity in primary and secondary prevention of cardiovascular disease: Overview updated. World J Cardiol 2016;8:575-83.
24. Karmisholt K, Gøtzsche PC. Physical activity for secondary prevention of disease. Systematic reviews of randomised clinical trials. Dan Med Bull 2005;52:90-4.

25. Research and Geospatial Information Team. Indices of Multiple Deprivation. 2015. Luton, 2015. Indices of deprivation in Luton.pdf. https://www.luton.gov.uk/Environment/Lists/LutonDocuments/PDF/ Planning/Observatory/2015. (accessed 5 Apr 2018).

26. Department for Communities and Local Government. The English Index of Multiple Deprivation (IMD) 2015- Guidance. 2015 https:// www.gov.uk/government/uploads/system/uploads/attachment data/file/464430/English_Index_of_Multiple_Deprivation_2015_Guidance.pdf (accessed 16 Mar 2018).

27. Scholes S, Bridges S, Ng Fat L, et al. Comparison of the Physical Activity and Sedentary Behaviour Assessment Questionnaire and the Short-Form International Physical Activity Questionnaire: An Analysis of Health Survey for England Data. PLoS One 2016;11:e0151647-30.

28. IPAQ Research Committee. Guidelines for Data Processing and Analysis of the International Physical Activity Questionnaire (IPAQ) - Short and Long Forms. 2005:1-15 https://www.scribd.com/doc/ 92677948/IPAQ-Scoring (accessed 12 May 2016).

29. Spadano JL, Must A, Bandini LG, et al. Energy cost of physical activities in 12-y-old girls: MET values and the influence of body weight. Int J Obes Relat Metab Disord 2003;27:1528-33.

30. Slymen DJ, Ayala GX, Arredondo EM, et al. A demonstration of modeling count data with an application to physical activity. Epidemiol Perspect Innov 2006;3:3:3.

31. Ghosh SK, Mukhopadhyay P, Lu Jye-Chyi(JC), J-c L. Bayesian analysis of zero-inflated regression models. J Stat Plan Inference 2006;136:1360-75 https://doi.org/.

32. Zyphur MJ, Oswald FL. Bayesian Estimation and Inference: A User's Guide. J Manage 2015;41:390-420.

33. Lynch SM, Western B. Bayesian Posterior Predictive Checks for Complex Models. Sociol Methods Res 2004;32:301-35.

34. Gelman A, Carlin J, Stern H, et al; Bayesian Data Analysis. 3rd ed. Boca Raton, FL: CRC Press Taylor \& Francis Group, 2013.

35. Hardin J, Hilbe J. Generalized Linear Models and Extensions. 3rd ed. College Station, TX: Stata Press, 2012.

36. Woodall J, Trigwell J, Bunyan AM, et al. Understanding the effectiveness and mechanisms of a social prescribing service: a mixed method analysis. BMC Health Serv Res 2018;18:1-12.

37. Ross R, Hill JO, Latimer A, et al. Evaluating a small change approach to preventing long term weight gain in overweight and obese adults--Study rationale, design, and methods. Contemp Clin Trials 2016;47:275-81.

38. Hills AP, Byrne NM, Lindstrom R, et al. 'Small changes' to diet and physical activity behaviors for weight management. Obes Facts 2013;6:228-38.

39. Basolo A, Votruba SB, Heinitz S, et al. Deviations in energy sensing predict long-term weight change in overweight Native Americans. Metabolism 2018;82:65-71.

40. White J, Kinsella K, South J. An evaluation of social prescribing health trainers in South and West Bradford. $2010 \mathrm{http}: / /$ www.involvey orkshirehumber.org.uk/uploads/files/workshop-presentations/socialprescribing-evaluation-and-health-trainers.pdf (accessed 19 Oct 2018).

41. Farenden C, Mitchell C, Feast S, et al. Community navigation in Brighton \& Hove. Evaluation of a social prescribing pilot 2015;68 http://www.bh-impetus.org/wp-content/uploads/2015/12/CN-FullEvaluation-Nov-2015.pdf.

42. Friedli L, Themessl-huber M, Butchart M. Evaluation of Dundee Equally Well sources of support: Social prescribing in Maryfield. 2012;42 https://www.northstaffsccg.nhs.uk/your-ccg/ ns-publications/generic-publications/1324-social-prescribingevaluation-dundee/file.

43. Moore G, Audrey S, Barker M, et al. Process evaluation of complex interventions. UK Medical Research Council (MRC) guidance 2014:1-133 http://decipher.uk.net/wp-content/uploads/2014/11/ MRC-PHSRN-Process-evaluation-guidance.pdf (accessed 18 Oct 2018).

44. Pescheny J, Randhawa G, Pappas Y. Patient uptake and adherence to social prescribing: a qualitative study. BJGP Open 2018;2:bjgpopen18X101598.

45. Barte JCM, Wendel-Vos GCW. A Systematic Review of Financial Incentives for Physical Activity: The Effects on Physical Activity and Related Outcomes. Behav Med 2017;43:79-90.

46. Milanović Z, Pantelić S, Trajković N, et al. Age-related decrease in physical activity and functional fitness among elderly men and women. Clin Interv Aging 2013;8:549-56. 
47. van de Schoot R, Kaplan D, Denissen J, et al. A gentle introduction to bayesian analysis: applications to developmental research. Child Dev 2014;85:842-60.

48. Berger JO. Statistical Decision Theory and Bayesian Analysis. 2nd ed. New York: Springer, 1993.

49. Ling T, Brereton L, Conklin A, et al. Barriers and facilitators to integrating care: experiences from the English Integrated Care Pilots. Int J Integr Care 2012;12:1-12.
50. Mackie S, Darvill A. Factors enabling implementation of integrated health and social care: a systematic review. Br J Community Nurs 2016;21:82-7.

51. Wigfield A, Kispeter E, Alden S, et al. Age UK's fit for the future Project: Evaluation Report. 2015 https://www.sheffield.ac.uk/ polopoly_fs/1.569567!/file/fit-for-the-future-Final-Evaluation.pdf (accessed 19 Oct 2018). 